\title{
Minimally invasive transforaminal lumbar interbody fusion: indications, technique, and complications
}

\author{
Langston T. Holly, M.D., James D. Schwender, M.D., David P. Rouben, M.D., \\ AND KeVIN T. Foley, M.D. \\ Division of Neurosurgery, David Geffen University of California at Los Angeles Medical Center, \\ Los Angeles, California; Twin Cities Spine Center and Department of Orthopedic Surgery, \\ University of Minnesota, Minneapolis, Minnesota; River City Orthopaedic Surgeons, \\ Louisville, Kentucky; and Semmes-Murphey Clinic and Department of Neurosurgery, \\ University of Tennessee, Memphis, Tennessee
}

\begin{abstract}
$\checkmark$ The authors provide an overview of the minimally invasive transforaminal lumbar interbody fusion (TLIF) procedure including indications, technique, and complications. This novel technique is a method of achieving circumferential lumbar fusion using a unilateral dorsal approach. Minimally invasive TLIF uses a tubular retractor that is inserted via a muscle-dilating exposure, thereby minimizing the approach-related morbidity. This procedure is ideal for refractory mechanical low-back and radicular pain associated with spondylolisthesis, degenerative disc disease, and recurrent disc herniation. The authors' clinical experience and review of the medical literature indicate that TLIF can be effectively and safely performed in a minimally invasive fashion.
\end{abstract}

\section{KEY WoRds • minimally invasive surgery • posterior lumbar interbody fusion • spinal fusion - transforaminal lumbar interbody fusion}

\section{$\mathrm{L}$}

UMBAR fusion has undergone significant evolution since Cloward ${ }^{2}$ first introduced the original PLIF procedure more than 50 years ago. Although PLIF is still commonly performed, $1,8,18,27$ many surgeons now prefer the TLIF procedure in the management of a variety of spinal disorders that require lumbar arthrodesis. ${ }^{16,19,24}$ This procedure offers some distinct advantages to PLIF, including unilateral exposure, decreased neural retraction, and a more lateral angle of approach that facilitates revision surgery.

The use of minimally invasive surgical techniques represents the most recent modification of methods used to achieve lumbar interbody fusion. Conventional lumbar fusion is associated with significant muscle stripping and retraction that can adversely affect both short- and longterm patient outcomes. ${ }^{9} 14,15,17,22,26,28$ In contrast, minimally invasive lumbar fusion is performed via a muscle-dilating approach and significantly diminishes the amount of iatrogenic soft-tissue injury. As a result, the new procedure has shown the potential to reduce the amount of intraoperative blood loss, the intensity of postoperative pain, and the duration of hospital stays. ${ }^{5,6,25}$

The minimally invasive TLIF procedure was first des-

Abbreviations used in this paper: $\mathrm{AP}=$ anteroposterior; $\mathrm{MR}=$ magnetic resonance; PLIF = posterior lumbar interbody fusion; TLIF $=$ transforaminal lumbar interbody fusion. cribed by Foley, et al., ${ }^{5}$ and it has since become an increasingly popular method of lumbar arthrodesis. . $2,13,20,21,25^{\text {In }}$ this study, we describe aspects of the procedure, including indications, surgical technique, and complications.

\section{Indications}

The surgical indications for minimally invasive TLIF closely mirror those of open TLIF and are, in part, a function of the individual surgeon's experience and comfort level with these procedures. Prior to attempting the minimally invasive TLIF procedure, adequate familiarity and competence with more straightforward minimal-access procedures such as microdiscectomy should be achieved. Furthermore, it is important for the surgeon to have acquired sufficient experience with the open TLIF procedure prior to attempting minimally invasive TLIF (Fig. 1).

As with other forms of lumbar fusion, the best clinical results are generally associated with single-level disease (Fig. 2), although two-level minimally invasive fusion can be performed in properly selected patients. One of the best indications for minimally invasive TLIF is the treatment of mechanical low-back and radicular pain associated with spondylolisthesis. Grade I or II spondylolisthesis is usually suitable for minimally invasive applications; highgrade slips are technically very challenging and are optimally treated by most surgeons via an open approach. Minimally invasive TLIF provides the ability to directly 
L. T. Holly, et al.

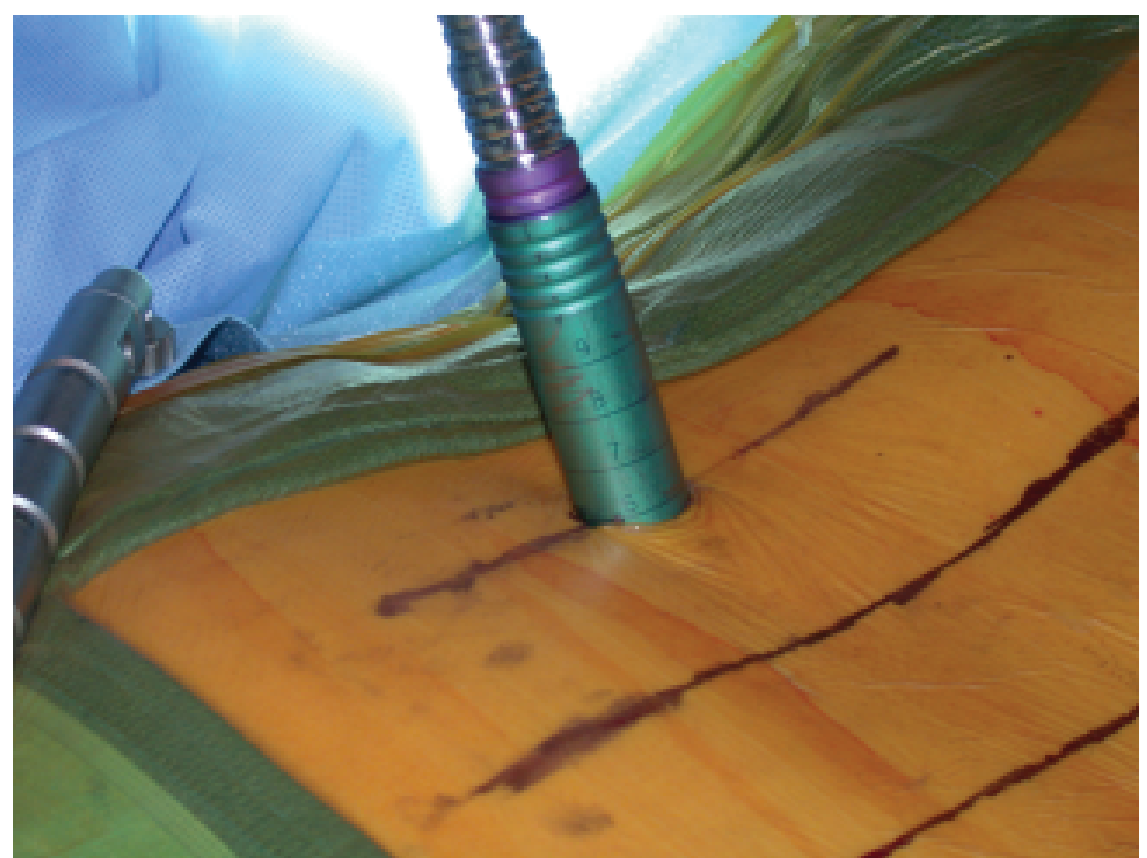

FIG. 1. Intraoperative photograph demonstrating placement of sequential tissue dilators prior to tubular retractor placement.

decompress both the ipsilateral exiting and traversing nerve roots. In cases of bilateral radiculopathy, contralateral decompression can be indirectly achieved following graft placement once foraminal height and sagittal balance have been restored. In patients with severe contralateral lateral recess stenosis, a contralateral minimally invasive decompression can be performed through a separate incision using a tubular retractor.

Patients who have suffered from recurrent disc herniation are also good candidates for the minimally invasive TLIF procedure. This can be the definitive treatment for patients with or without low-back pain who have suffered multiple recurrent disc herniations. Patients with only one recurrence but severe mechanical low-back pain can be considered for this procedure as well. It can be performed on the same side as the original surgery because the transforaminal approach is usually lateral to the scar tissue. Other potential indications for TLIF include severe discogenic low-back pain caused by degenerative disc disease, postlaminectomy instability, spinal trauma, or the treatment of pseudarthrosis.

One of the few contraindications for this procedure is the presence of a conjoined nerve root within the foramen. Although this is an extremely rare occurrence, preoperative MR images should be closely inspected for this phenomenon. In such cases, the conjoined root lies directly over the desired entry point, and attempts to mobilize the neural elements have an unacceptable rate of neurological injury. If the presence of the conjoined root is determined only after the facetectomy has been completed, the procedure should be aborted. At that point, a contralateral TLIF could be considered or the procedure could be converted to a minimally invasive posterolateral fusion.

\section{Surgical Technique}

Following the induction of general endotracheal anesthesia, the patient is carefully turned to the prone position on a radiolucent table. Options include using a standard table with chest rolls to help maintain the lumbar lordosis or a Jackson-type table that places the hips in extension. All of the pressure points are appropriately padded. Prior to preparing and draping the patient, lateral and AP fluoroscopic images are obtained to make sure that the pedicles and other relevant spinal anatomy can be identified. After the patient is draped, a 22-gauge spinal needle is inserted into the skin, directly over the disc space of interest. A $2.5-\mathrm{cm}$ skin incision is centered over this point, approximately 4 to $5 \mathrm{~cm}$ lateral to the midline. The side of the approach is based on the location of the preoperative radicular symptoms. Hemostasis is achieved with electrocautery. Then, using fluoroscopic guidance, a K-wire is directed through the incision toward the facet complex with a lateral-to-medial trajectory. The wire is passed through only the fascia and muscle to avoid the risk of inadvertent neurological injury or dural puncture. Serial dilators are passed over the wire, which creates a muscle-splitting surgical channel. The appropriate-length tubular retractor (22or 26-mm-diameter METRx, or the Quadrant; Medtronic Sofamor Danek, Memphis, TN) is passed over the dilators and centered over the facet joint. The retractor is then attached to the table frame via a flexible articulated arm. The remainder of the procedure can be performed using an operating microscope or an illuminated tube and loupe magnification, depending on the surgeon's preference.

Residual soft tissue is removed from the dorsal surface of the facet complex using electrocautery and pituitary 

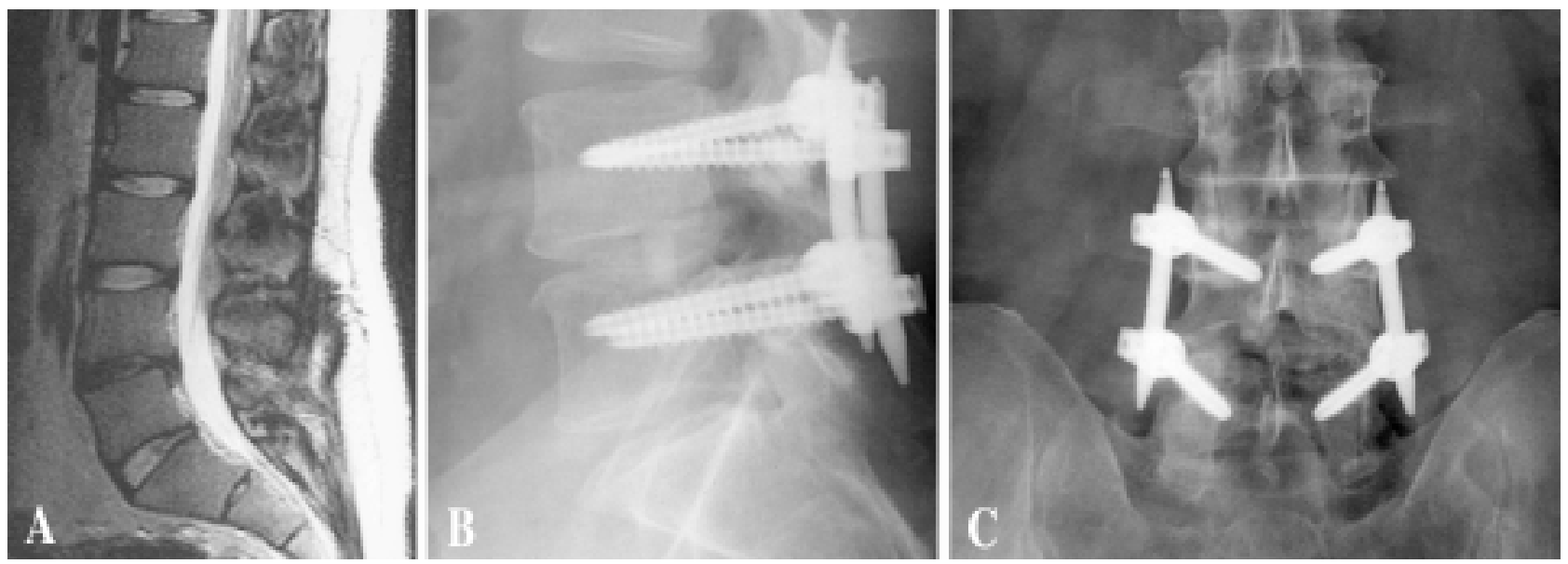

FIG. 2. A: Sagittal MR image obtained in a 45-year-old man with refractory low-back pain radiating into his leg in the L-5 distribution. B and C: Postoperative lateral and AP radiographs obtained 6 months following surgery, showing successful arthrodesis.

instruments. A total facetectomy is performed using bayoneted osteotomes or the high-speed drill. An initial cut is made across the medial aspect of the inferior facet, disarticulating it from the superior lamina. It is then carefully separated from the synovial membrane and the ligamentum flavum by using curved curettes; these tissues are in continuity with each other and form one plane in the lateral recess. The inferior facet is then removed from the field using a pituitary instrument. The remaining superior facet is then partially drilled and removed in piecemeal fashion using a Kerrison rongeur. Care must be taken to avoid breeching the inferior pedicle while using the highspeed drill. All of the removed bone is morcellized and saved for use as graft material later in the case. A carpet of ligamentum flavum and synovial membrane is left completely intact to serve as a protective barrier for the dura mater and nerve roots. The ligamentum flavum and synovium are now removed in piecemeal fashion using Kerrison instruments to expose the exiting and traversing nerve roots. Epidural bleeding is commonly encountered at this point and is easily controlled using thrombin-soaked Gelfoam and bipolar cautery. It is not necessary to expose the entire exiting nerve root, but the surgeon should be aware of both the root's location beneath the superior pedicle and the amount of space between the lateral margin of the traversing root and the medial edge of the exiting root. In patients with symptomatic central stenosis, a more medial laminotomy can be performed by angling the tubular retractor. In fact, bilateral decompression can be achieved in this fashion by undercutting the spinous process and drilling away the contralateral lamina.

A standard ipsilateral discectomy is performed through the tubular retractor. Not uncommonly, posterior osteophytes will need to be removed to optimize access to the disc space. This can be achieved using a Kerrison rongeur or a bayoneted osteotome. The interspace is then distracted using one of two methods. In the first method, a $2.5-\mathrm{cm}$ "mirror image" skin incision is created on the contralateral side, centered over the interspace, and percutaneous Sextant screws (Medtronic Sofamor Danek) and a rod are placed to distract the interspace and temporarily maintain the distracted position (Fig. 3) ${ }^{3,4}$ In the second method, an interlaminar spreader is placed through the incision to a location between the spinous processes and used for interspace distraction. Further interspace distraction is achieved using customized intervertebral body spreaders. Distraction is maintained if needed by tightening the contralateral screws in the distracted position. Once the optimal interspace distraction has been achieved, endplate preparation is performed using custom-designed downpushing curettes and endplate scrapers. All of the cartilaginous endplate is removed, and the bone endplates are decorticated but left structurally intact. In most cases neural retraction is not necessary, but a small retractor often is placed over the traversing nerve root while instruments are being exchanged within the disc space. On occasion, the exiting nerve root may need to be slightly elevated at the L5-S1 level, depending on its angle of descent.

Based on the surgeon's preference, local autologous bone, structural allograft bone, interbody cages, and/or bone morphogenetic protein can be placed in the interspace. Local autograft bone and the sponge carrying bone morphogenetic protein are placed contralateral to the anulotomy in the anterior portion of the interspace. After the structural graft is placed, a nerve hook is passed beneath the neural elements to check for any evidence of compression. Ipsilateral percutaneous screws and a rod are then placed through the same incision. The screw/rod construct is gently compressed, thereby recreating lordosis as well as providing compression for the bone graft within the interspace. Contralateral percutaneous pedicle screws are now placed if this was not done during the distraction stage.

\section{Complications}

Small cerebrospinal fluid leaks can be treated with collagen sponge, fibrin glue, and flat bedrest for 24 hours. Primary dural closure is technically difficult and not necessary in the vast majority of cases. The risk of screw misplacement can be minimized by obtaining high quality AP and lateral fluoroscopic images. To avoid parallax, the 


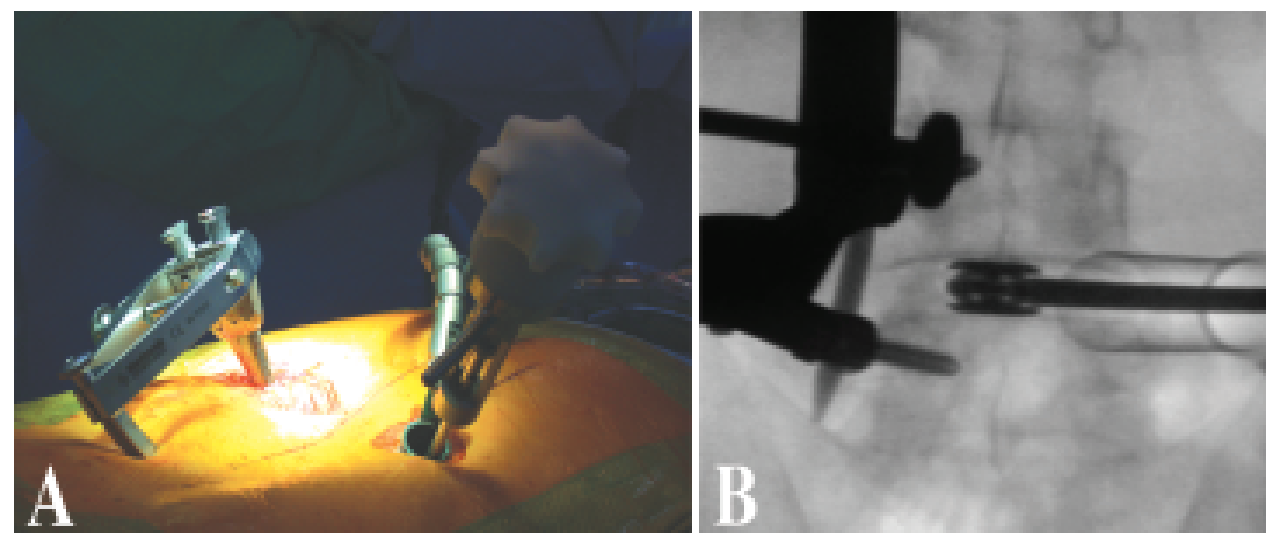

FIG. 3. Intraoperative photograph (A) and fluoroscopic image (B) showing interbody distractor in place while percutaneous screws are being placed on the contralateral side.

region of interest should be in the center of the fluoroscopic view. Alternatively, image-guidance techniques can be used to facilitate percutaneous screw placement. Interbody grafts should be countersunk in the intervertebral space to reduce the chance of migration through the opening in the anulus.

\section{Discussion}

\section{Transforaminal Lumbar Interbody Fusion}

The TLIF procedure was first introduced by Harms and colleagues $^{10,11}$ as an alternative to PLIF for the management of a variety of spinal disorders that require lumbar fusion. This procedure offers many of the same advantages as PLIF, such as the ability simultaneously to decompress the neural elements, correct abnormalities in alignment and sagittal balance, and achieve circumferential arthrodesis through a single dorsal approach. Both TLIF and PLIF yield high arthrodesis rates secondary to a large fusion surface area, ample blood supply to the interbody space, and the ability to place the interbody graft in compression. However, the more lateral to medial approach offered by TLIF has several potential advantages over PLIF. Because access is obtained via a transforaminal approach with TLIF, little if any neural retraction is required to enter the disc space. In contrast, PLIF almost always requires neural manipulation and retraction, and neurological injury is one of the most commonly reported complications of that procedure. ${ }^{23}$ Furthermore, revision surgery with TLIF is simplified because the angle of approach avoids midline scar tissue and uses previously undisturbed tissue planes. Also, TLIF procedures are performed unilaterally (obviating the need for bilateral epidural space exposure), so they are frequently associated with shorter operating room times.

\section{Rationale for Minimally Invasive Fusion}

Conventional lumbar fusion surgery is associated with iatrogenic soft-tissue injury that can have deleterious shortand long-term effects on patient outcome. Numerous studies have demonstrated that irreversible muscle injury occurs as a result of muscle stripping and retraction and can be associated with poor clinical results. ${ }^{914,15,17,26,28}$ Gejo, et al., ${ }^{9}$ prospectively evaluated serial changes in trunk muscle performance in 20 patients who underwent posterior lumbar surgery. The patients were divided into short $(<80$ minutes) and long ( $>80$ minutes) retraction-time groups. The degree of back muscle injury was estimated by comparing preoperative and postoperative MR images. Muscle strength was evaluated during trunk flexion and extension by using a kinetic measuring device and dynamometer. The authors determined that damage to the multifidus muscle was more severe, and the recovery of extensor muscle strength delayed, in the long retraction-time group. Sihvonen, et al., ${ }^{26}$ studied a group of patients with severe postoperative failed-back surgery syndrome, using radiological and neurophysiological studies as well as muscle biopsy prodecures. They concluded that disrupted back-muscle innervation and loss of muscular support could be an important factor in the development of failed-back surgery syndrome. They suggested the potential role of developing surgical methods that preserve back muscle innervation better than conventional techniques.

The goal of any minimally invasive procedure is to achieve the same surgical objectives as the corresponding open procedure through a less traumatic approach. It is believed that these less invasive approaches will result in decreased intraoperative blood loss, less postoperative pain, and shorter hospital stays without compromising the efficacy of the procedure. Minimally invasive TLIF uses a tubular retractor system and modified surgical instruments that were first developed for the minimally invasive microdiscectomy procedure. ${ }^{7}$ This technology provides a paramedian, muscle-dilating approach that maintains the normal midline musculoskeletal structures. The small working corridor is in stark contrast to the large midline incision and soft-tissue exposure commonly performed with open TLIF.

\section{Benefits of Minimally Invasive TLIF}

A number of recently published manuscripts, with some modifications of the originally described procedure, have detailed the apparent benefits of this novel technique. . $^{12,13,}$ ${ }^{20,21,25}$ Schwender, et al., ${ }^{25}$ affirmed the feasibility of safely and effectively performing the TLIF procedure through a minimally invasive approach in a series of 49 patients with a mean of 22.6 months of follow up. All 49 patients 
noted significant improvement in their low-back pain following the surgery. Radicular pain resolved in all patients postoperatively as well. Scores for the patients on the visual analog scale and the Oswestry Disability Index improved from 7.2 to 2.1 and 46 to 21 , respectively. The mean hospital stay was 1.9 days, and the average estimated blood loss was $140 \mathrm{ml}$. Jang and Lee ${ }^{13}$ also reported significant improvements in postoperative visual analog scale and Oswestry Disability Index scores in a group of 23 patients who underwent minimally invasive TLIF using ipsilateral pedicle screw and contralateral facet screw fixation. Isaacs, et al., ${ }^{12}$ compared a series of 20 patients who underwent endoscopically assisted minimally invasive TLIF with a group of patients who underwent the open PLIF procedure for single-level degenerative disease. They determined that intraoperative blood loss, hospital stay, and postoperative narcotic use were significantly less in the patients who underwent minimally invasive TLIF procedures. Mummaneni and Rodts ${ }^{20}$ described the miniopen TLIF technique, in which an expandable tubular retractor is used in a Wiltse-type approach. This technique provides direct simultaneous visualization of both pedicles and allows placement of cannulated or noncannulated screws through the expandable retractor. The contralateral screws can be placed percutaneously or by using another mini-open approach.

\section{Conclusions}

The TLIF procedure can be safely and effectively performed in a minimally invasive fashion. Preliminary results suggest at least equivalent clinical outcomes compared with conventionally performed open TLIF. Postoperative pain, intraoperative blood loss, and hospital stays appear to be significantly less with the minimally invasive approach. As with all novel techniques, there is a learning curve that can be overcome with experience and repetition.

\section{References}

1. Branch CL: The case for posterior lumbar interbody fusion. Clin Neurosurg 43:252-267, 1996

2. Cloward RB: The treatment of ruptured intervertebral discs by vertebral body fusion. I. Indications, operative technique, after care. J Neurosurg 10:154-168, 1953

3. Foley KT, Gupta SK: Percutaneous pedicle screw fixation of the lumbar spine: preliminary clinical results. J Neurosurg 97 (1 Suppl):7-12, 2002

4. Foley KT, Gupta SK: Percutaneous pedicle screw fixation of the lumbar spine: preliminary clinical results. Neurosurg Focus 10(4):E10, 2001

5. Foley KT, Holly LT, Schwender JD: Minimally invasive lumbar fusion. Spine 28:S26-S35, 2003

6. Foley KT, Lefkowitz MA: Advances in minimally invasive spine surgery. Clin Neurosurg 49:499-517, 2002

7. Foley KT, Smith MM: Microendoscopic discectomy. Tech Neurosurg 3:301-307, 1997

8. Fraser RD: Interbody, posterior, and combined lumbar fusions. Spine 20:S167-S177, 1995

9. Gejo R, Matsui H, Kawaguchi Y, et al: Serial changes in trunk muscle performance after posterior lumbar surgery. Spine 24: 1023-1028, 1999
10. Harms JG, Jeszenszky D: The unilateral transforaminal approach for posteriorlumbar interbody fusion. Orthop Traumatol 6:88-99, 1998

11. Harms JG, Rollinger H: Die operative Behandlung der Spondylolisthese durch dorsale Aufrichtung und ventrale Verblockung. Z Orthop 120:343-347, 1982

12. Isaacs RE, Podichetty VK, Santiago P, et al: Minimally invasive microendoscopy-assisted transforaminal interbody fusion with instrumentation. J Neurosurg Spine 3:98-105, 2005

13. Jang JS, Lee SH: Minimally invasive transforaminal lumbar interbody fusion with ipsilateral pedicle screw and contralateral facet screw fixation. J Neurosurg Spine 3:218-223, 2005

14. Kawaguchi Y, Matsui H, Tsuji H: Back muscle injury after posterior lumbar spine surgery. A histologic and enzymatic analysis. Spine 21:941-944, 1996

15. Kawaguchi Y, Matsui H, Tsuji H: Back muscle injury after posterior lumbar spine surgery. Part 2: Histologic and histochemical analyses in humans. Spine 19:2598-2602, 1994

16. Lowe TG, Tahernia AD, O'Brien MF, et al: Unilateral transforaminal posterior lumbar interbody fusion (TLIF): indications, technique, and 2-year results. J Spinal Disord Tech 15:31-38, 2002

17. Mayer TG, Vanharanta H, Gatchel RJ, et al: Comparison of CT scan muscle measurements and isokinetic trunk strength in postoperative patients. Spine 14:33-36, 1989

18. McLaughlin MR, Haid RW Jr, Rodts GE, et al: Posterior lumbar interbody fusion: indications, techniques, and results. Clin Neurosurg:514-527, 2000

19. Moskowitz A: Transforaminal lumbar interbody fusion. Orthop Clin North Am 33:359-366, 2002

20. Mummaneni PV, Rodts GE Jr: The mini-open transforaminal lumbar interbody fusion. Neurosurgery 57: 256-261, 2005

21. Ozgur BM, Yoo K, Rodriguez B, et al: Minimally-invasive technique for transforaminal lumbar interbody fusion (TLIF). Eur Spine J 14:887-894, 2005

22. Rantanen J, Hurme M, Falck B, et al: The lumbar multifidus muscle five years after surgery for a lumbar intervertebral disc herniation. Spine 18:568-574, 1993

23. Ray CD: Threaded titanium cages for lumbar interbody fusions. Spine 22:667-680, 1997

24. Rosenberg WS, Mummaneni PV: Transforaminal lumbar interbody fusion: technique, complications, and early results. Neurosurgery 48:569-575, 2001

25. Schwender JD, Holly LT, Rouben DP, et al: Minimally invasive transforaminal lumbar interbody fusion (TLIF): technical feasibility and initial results. J Spinal Disord Tech 18 (Suppl 1): S1-S6, 2005

26. Sihvonen T, Herno A, Paljarvi L, et al: Local denervation atrophy of paraspinal muscles in postoperative failed back syndrome. Spine 18:575-581, 1993

27. Stonecipher T, Wright S: Posterior lumbar interbody fusion with facet-screw fixation. Spine 14:468-471, 1989

28. Styf JR, Willen J: The effects of external compression by three different retractors on pressure in the erector spine muscles during and after posterior lumbar spine surgery in humans. Spine 23:354-358, 1998

Manuscript received January 17, 2006.

Accepted in final form February 7, 2006.

Address reprint requests to: Langston T. Holly, M.D., 74-144 CHS, 10833 Le Conte Avenue, David Geffen University of California at Los Angeles School of Medicine, Los Angeles, California 90095. email: 1holly@ mednet.ucla.edu. 